\title{
Jet-CO alignments in the environments high-z radio galaxies
}

\author{
Bjorn Emonts \\ Centro de Astrobiología (CSIC-INTA), Ctra de Torrejón a Ajalvir, km 4, 28850 \\ Torrejón de Ardoz, Madrid, Spain
}

\begin{abstract}
In the outskirts of massive high-redshift radio galaxies, powerful radio-jets often interact with ambient warm Ly $\alpha$-emitting gas. We present the discovery of luminous reservoirs of cold molecular gas in these environments, based on $\mathrm{CO}(1-0)$ observations with the Australia Telescope Compact Array. The CO-emission is aligned with the radio jets, and found tens of kpc outside the host galaxy. These molecular gas reservoirs have $\mathrm{CO}$ luminosities in the range of those found in submm-galaxies $\left(L_{\mathrm{CO}}^{\prime} \sim 4-9 \times 10^{10} \mathrm{~K} \mathrm{~km} / \mathrm{s} \mathrm{pc}^{2}\right)$, but they lack any near-infrared counterpart in deep Spitzer imaging. These results suggest that jet-triggered feedback takes place in the circum-galactic environment of high- $z$ radio galaxies. We prefer the interpretation that the CO-emitting gas is formed when the propagating jets enrich, shock and cool pre-existing dusty halo gas. We further argue that sensitive low-surface-brightness CO observations, using radio interferometers in very compact array-configurations, are essential to study the role of the cold molecular medium in the outskirts of massive high- $z$ galaxies.
\end{abstract}

Keywords. galaxies: halos, active, jets, intergalactic medium, techniques: interferometric

\section{Introduction}

High-z radio galaxies (HzRGs) are among the most massive and active galaxies in the Universe (e.g. Miley \& De Breuck 2008). They are often embedded in giant $\sim 100 \mathrm{kpc}$ halos of Ly $\alpha$-emitting gas (T $\sim 10^{4} \mathrm{~K}$; Villar-Martin et al. 2003). Soon after their initial discovery in the late 1980s, near-universal alignments were found between the radio synchrotron jets and the optical/UV morphology of the host galaxies (Chambers et al. 1987; Mc Carthy et al. 1987). These alignments were initially ascribed to jet-triggered star-formation, although their true nature has since remained elusive. Later, similar alignments were reported between the radio jets and the distribution of both dust and cold molecular gas (Stevens et al. 2003, Klamer et al. 2004).

We here report on recently completed $\mathrm{CO}(1-0)$ studies, which reveal that detectable amounts of cold molecular gas can reside in the environments of HzRGs (Emonts et al. 2014, 2015a,b). We confirm the trend that CO often aligns with the radio jets, as evidence for jet-induced feedback onto the gaseous medium around HzRGs,

\section{Methods}

We used the 30-50 GHz system of the Australia Telescope Compact Array (ATCA) to study $\mathrm{CO}(1-0)$ in a representative sample of 13 southern HzRGs at $z \sim 1.4-2.9$, with no selection-bias on infrared- or submm-luminosity (Emonts et al. 2014). The on-source integration time ranged from $10-25 \mathrm{~h}$ per target. The ground-transition $\mathrm{CO}(1-0)$ is the most robust tracer of the overall molecular gas mass, including any widespread and subthermally excited component (Papadopoulos et al. 2001). The ATCA has very compact, hybrid array-configurations with short baselines, which makes it sensitive for detecting low-surface-brightness emission of $\mathrm{CO}(1-0)$. 

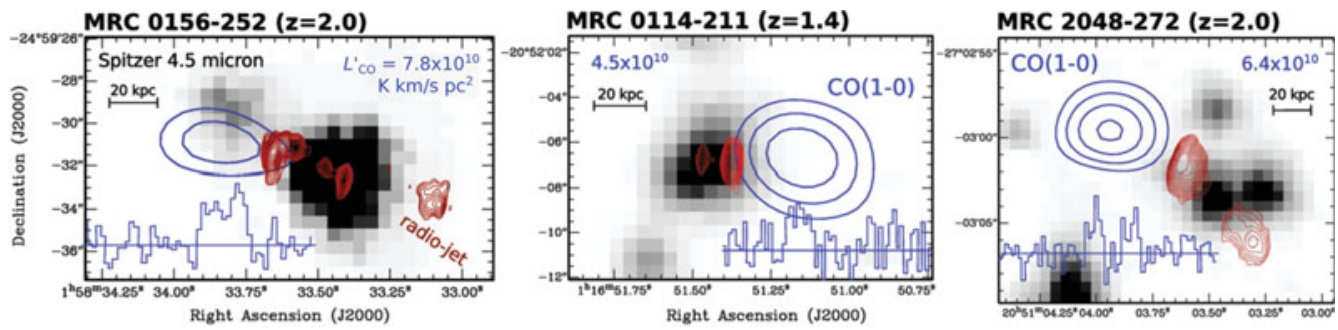

Figure 1. $\mathrm{CO}(1-0)$ in the environments of three HzRGs (adapted from Emonts et al. 2014, their Fig. 5). The CO (thick blue contours) is found beyond the radio-jet (thin red contours), at a location devoid of Spitzer $4.5 \mu \mathrm{m}$ emission (greyscale). CO contours: $2.8,3.5,4.2,4.9 \sigma$, with $\sigma=0.095,0.094,0.080 \mathrm{Jy} /$ beam $\times \mathrm{km} / \mathrm{s}$ for MRC 0156-252, 0114-211, 2048-272. The $8.2 \mathrm{GHz}$ radio-continuum data are from Carilli et al. (1997) and De Breuck et al. (2010), Spitzer data from Wylezalek et al. (2013); color version available online.

\section{Results}

We detect $\mathrm{CO}(1-0)$ in 5 out of 13 HzRGs (Emonts et al. 2014). Fig. 1 shows three cases where an unresolved $\mathrm{CO}(1-0)$ reservoir is found tens of $\mathrm{kpc}$ outside the radio galaxy. The $\mathrm{CO}(1-0)$ is located beyond the brightest edge or the radio source, and covers FWZI $_{\mathrm{CO}} \sim 1000-2000 \mathrm{~km} / \mathrm{s}$. The CO luminosities, $L_{\mathrm{CO}}^{\prime} \sim 4-9 \times 10^{10} \mathrm{~K} \mathrm{~km} / \mathrm{s} \mathrm{pc}^{2}$, are comparable to those of submm galaxies, but no counterpart is visible in deep $4.6 \mu \mathrm{m}$ Spitzer data. This is similar to the case of TXS 0828+193 (Nesvadba et al. 2009). Therefore, the $\mathrm{CO}$ traces a cold and possibly diffuse molecular medium.

Fig. 2 shows the CO-rich environment of the Dragonfly Galaxy (MRC 0152-209) at $z=1.92$. This is one of the most IR-luminous HzRGs $\left(\mathrm{SFR} \sim 3000 \mathrm{M}_{\odot} \mathrm{yr}^{-1}\right.$; Drouart et al. 2014). ALMA revealed three compact $\mathrm{CO}(6-5)$ components in the central $10 \mathrm{kpc}$, classifying this is a rare triple merger (Emonts et al. 2015b). With ATCA we detect tidal debris in $\mathrm{CO}(1-0)$ across $\sim 60 \mathrm{kpc}$ (Emonts et al. 2015a). Also here, the $\sim 18 \mathrm{kpc}$ radio source aligns with part of the extended CO.

\section{Discussion}

The jet-CO alignments suggest that powerful high- $z$ radio jets exert feedback onto the circum-galactic environment, tens of kpc outside the host galaxy. We believe that the molecular CO-emitting gas is likely formed when the propagating jets shock, compress and cool pre-existing dusty halo-gas (see discussion in Emonts et al. 2015a). Possibly the jets also enrich the gas by dragging metals out into the environment (Kirkpatrick et al. 2011). These $\mathrm{CO}(1-0)$ results may thus provide evidence in support of predicted key processes that drive the early evolution of massive galaxies, such as jet-triggered star formation or cooling flows. However, the exact mechanism behind the jet-CO alignments is not yet clear. An alternative explanation is that the radio jets brighten when they encounter a pre-existing reservoir of dense gas (also Stevens et al. 2003). We are currently analysing low-surface-brightness ALMA data to further study these cold gas reservoirs.

\section{Outlook}

Our results show the need to study the cold molecular medium around active galaxies to fully understand black-hole feedback in the Early Universe. Traditional mm-facilities can only target the $J \geqslant 3$ transitions of $\mathrm{CO}(J, J-1)$ at high- $z$. This led to a bias of tracing dense molecular gas in starburst/AGN regions. The advantage of targeting $\mathrm{CO}(1-0)$ at $\nu_{\mathrm{obs}}<50 \mathrm{GHz}$ is shown in Fig. 2: where ALMA reveals high- $J$ CO on kpc scales, ATCA -a 5 -dish interferometer at sea level- detects extended emission from the intrinsically much 


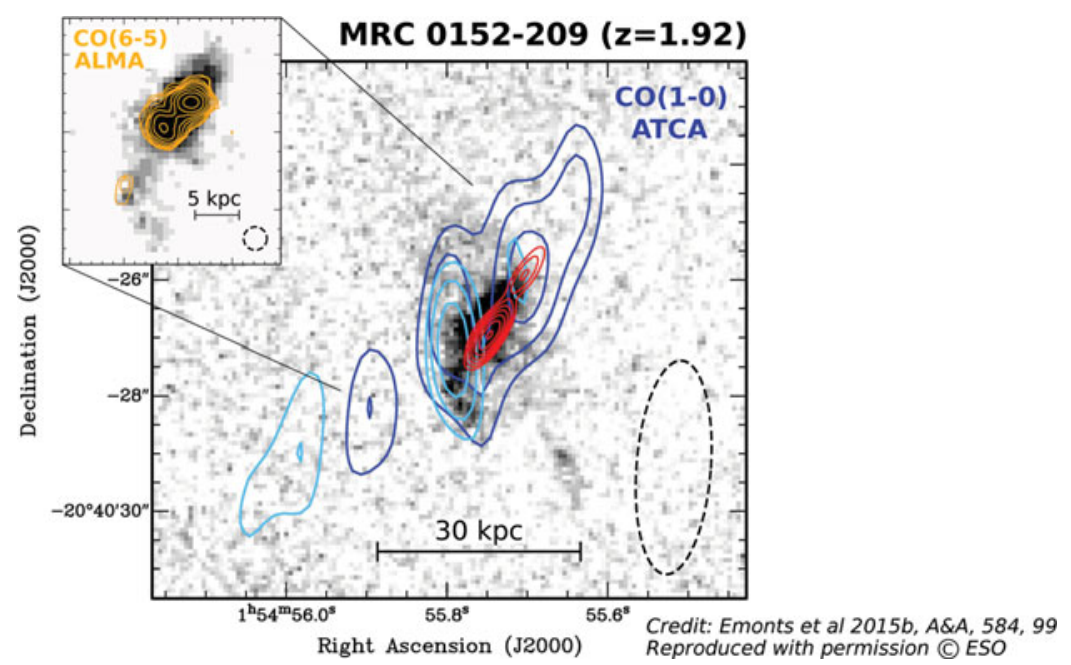

Figure 2. Molecular debris around the merging Dragonfly Galaxy (adapted from Emonts et al. $2015 \mathrm{a}, \mathrm{b})$. The thick dark (light) blue contours show the $\mathrm{CO}(1-0)$ in the velocity range $53-113$ $(113-172) \mathrm{km} / \mathrm{s}$, while the bulk of the $\mathrm{CO}(1-0)$ that coincides with the central galaxy has been omitted from this plot. The radio jet is shown in thin red contours. The grey-scale image is from HST/NICMOS (Pentericci et al. 2000, 2001). The inset shows CO(6-5) contours from ALMA. Contours: $\mathrm{CO}(1-0)-0.020,0.026,0.031 ; \mathrm{CO}(6-5)-0.1$ to $1.7($ steps $\times 1.4) \mathrm{Jy} / \mathrm{beam} \times \mathrm{km} / \mathrm{s}$.

weaker $\mathrm{CO}(1-0)$ across many tens of kpc. The key to finding this weak but extended $\mathrm{CO}$ is to use interferometers that are optimized for detecting low-surface-brightness emission, with as many short baselines as possible. Our ATCA results show the potential for lowsurface-brightness studies of the cold molecular medium when using very compact arrayconfigurations on future instruments, like ALMA/ACA Band 1 and next-generation VLA. Acknowledgments I thank my collaborators for their continuous help, and the organisers for a wonderful conference. This research has been funded by the European Union 7 th Framework Program (FP7-PEOPLE-2013-IEF) grant 624351. The Australia Telescope is funded by the Commonwealth of Australia as a National Facility managed by CSIRO.

\section{References}

Carilli, C. L., Röttgering, H. J. A., van Ojik, R., et al. 1997, ApJS, 109, 1

Chambers, K. C., Miley, G. K., \& van Breugel, W. 1987, Nature, 329, 604

De Breuck, C., Seymour, N., Stern, D., et al. 2010, ApJ, 725, 36

Drouart, G., De Breuck, C., Vernet, J., et al. 2014, A\&AA, 566, A53

Emonts, B. H. C., Norris, R. P., Feain, I., et al. 2014, MNRAS, 438, 2898

Emonts, B. H. C., Mao, M. Y., Stroe, A., et al. 2015a, MNRAS, 451, 1025

Emonts, B. H. C., De Breuck, C., Lehnert, M. D., et al. 2015b, A\&\&A, 584, A99

Kirkpatrick, C. C., McNamara, B. R., \& Cavagnolo, K. W. 2011, ApJL, 731, L23

Klamer, I. J., Ekers, R. D., Sadler, E. M., \& Hunstead, R. W. 2004, ApJL, 612, L97

McCarthy, P. J., van Breugel, W., Spinrad, H., \& Djorgovski, S. 1987, ApJL, 321, L29

Miley, G. \& De Breuck, C. 2008, A\&ARv, 15, 67

Nesvadba, N. P. H., Neri, R., De Breuck, C., et al. 2009, MNRAS, 395, L16

Papadopoulos, P., Ivison, R., Carilli, C., \& Lewis, G. 2001, Nature, 409, 58

Pentericci, L., Van Reeven, W., Carilli, C. L., et al. 2000, Aछ AS, 145, 121

Pentericci, L., McCarthy, P. J., Röttgering, H. J. A., et al. 2001, ApJS, 135, 63

Stevens, J. A., Ivison, R. J., Dunlop, J. S., et al. 2003, Nature, 425, 264

Villar-Martín, M., Vernet, J., di Serego Alighieri, S., et al. 2003, MNRAS, 346, 273

Wylezalek, D., Galametz, A., Stern, D., et al. 2013, ApJ, 769, 79 